\title{
A NOTE ON THE SUMMABILITY OF INFINITE SERIES BY SEQUENCE TO SEQUENCE AND SERIES TO SEQUENCE TRANSFORMATIONS
}

\author{
O. W. RECHARD
}

An infinite series $\sum_{n=1}^{\infty} t_{n}$ with partial sums $T_{n}$ is said to be summed to $T$ by the sequence to sequence transformation $A=\left(a_{i j}\right)$ in case $\lim _{i \rightarrow \infty} \sum_{j=1}^{\infty} a_{i j} T_{j}=T$. It is said to be summed to $T$ by the series to sequence transformation $B=\left(b_{i j}\right)$ in case $\lim _{i \rightarrow \infty} \sum_{j=1}^{\infty} b_{i j} t_{j}$ $=T$. A sequence to sequence or series to sequence transformation is regular if it sums every convergent series to its ordinary sum.

It is well known that corresponding to every regular sequence to sequence transformation $A=\left(a_{i j}\right)$ it is possible to construct a regular series to sequence transformation $B=\left(b_{i j}\right)$ by defining $b_{i j}=\sum_{n=1}^{\infty} a_{i n}$; moreover a series with bounded partial sums is summed by $A$ to $T$ if and only if it is summed by $B$ to $T$. The requirement that the series have bounded partial sums is essential, as is shown by the following example:

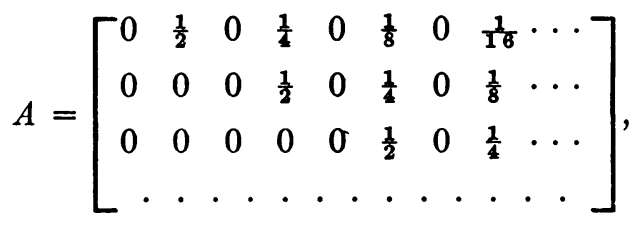

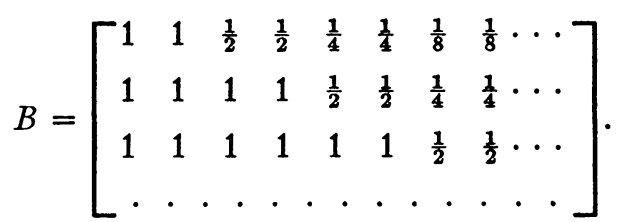

Both transformations $A$ and $B$ are regular, but the series $\sum t_{n}=2$ $-2+4-4+8-8+\cdots$ is summed by $A$ to 0 whereas none of the series $\sum_{j=1}^{\infty} b_{i j} t_{j}$ converge.

The process described in the preceding pargraph is not reversible however; that is, if $B=\left(b_{i j}\right)$ is a regular series to sequence transformation and $a_{i j}$ is defined by $a_{i j}=b_{i j}-b_{i, j+1}$, then the transformation $A=\left(a_{i j}\right)$ need not even be regular. For example, if each $b_{i j}=1$, then each $a_{i j}=0$.

It would thus appear that the series to sequence transformations are in some sense superior to the sequence to sequence transforma-

Received by the editors October 30, 1950. 
tions as means of summing divergent series. ${ }^{1}$ The purpose of this note is to point out that this superiority is really illusory. In fact, we shall prove the following theorem.

THEOREM. If $B=\left(b_{i j}\right)$ is a regular series to sequence transformation which sums some divergent series with bounded partial sums, and if $a_{i j}=b_{i j}-b_{i, j+1}$, then the transformation $A=\left(a_{i j}\right)$ is regular, and $a d i$ vergent series with bounded partial sums is summed by $B$ to $T$ if and only if it is summed by $A$ to $T$.

For suppose $\sum_{n=1}^{\infty} t_{n}$ is a divergent series with bounded partial sums $T_{n}$ which is summed by $B$ to $T$. Then, applying Abel's partial summation formula, we have for each $i$,

$$
\sum_{j=1}^{n} b_{i j} t_{j}=\sum_{j=1}^{n}\left(b_{i j}-b_{i, j+1}\right) T_{j}+b_{i, n+1} T_{n}=\sum_{j=1}^{n} a_{i j} T_{j}+b_{i, n+1} T_{n} .
$$

From the fact that $B$ is regular, it follows that $\sum_{j=1}^{\infty}\left|b_{i j}-b_{i, j+1}\right|$ $=\sum_{j=1}^{\infty}\left|a_{i j}\right|$ converges, ${ }^{2}$ and hence $\lim _{n \rightarrow \infty} b_{i n}$ exists. Also, since the $T_{j}$ are bounded, $\lim _{n \rightarrow \infty} \sum_{j=1}^{n} a_{i j} T_{j}$ exists, and, of course, $\lim _{n \rightarrow \infty}$ $\sum_{j=1}^{n} b_{i j} t_{j}$ exists. Consequently, $\lim _{n \rightarrow \infty} b_{i, n+1} T_{n}$ exists. If we assume that $\lim _{n \rightarrow \infty} b_{i n} \neq 0$, we are led to the contradictory conclusion that $\lim T_{n}$ exists, so we must conclude that $\lim _{n \rightarrow \infty} b_{i n}=0$.

Now if $\sum_{n=1}^{\infty} u_{n}$ is any series (convergent or divergent) with bounded partial sums $U_{n}$, then for each $i, \sum_{j=1}^{\infty} b_{i j} u_{j}=\sum_{j=1}^{\infty} a_{i j} U_{j}$. Hence the sequence into which $B$ transforms the series $\sum_{n=1}^{\infty} u_{n}$ is identical to the sequence into which $A$ transforms the sequence $\left\{U_{n}\right\}$, and the theorem follows.

\section{Los Alamos Scientific Laboratory}

${ }^{1}$ In this connection, see, for example, the preface to the book by C. N. Moore, Summable series and convergence factors, Amer. Math. Soc. Colloquium Publications, vol. 22, New York, 1938.

2 See, for example, Hardy, Divergent series, p. 51. 